\title{
Early Assessment of Delirium in Elderly Patients after Hip Surgery
}

\author{
Hyo Jin Lee ${ }^{1}$, Deuk Soo Hwang ${ }^{2}$, Seong Keun Wang${ }^{1}$, Ik Seung Chee ${ }^{1}$, Sengmi Baeg ${ }^{1}$ and Jeong Lan Kim ${ }^{1 凶}$ \\ ${ }^{1}$ Department of Psychiatry, School of Medicine, Chungnam National University, Daejeon, Korea \\ ${ }^{2}$ Department of Orthopedic Surgery, School of Medicine, Chungnam National University, Daejeon, Korea
}

\begin{abstract}
Objective This study is intended to identify predictive factors of delirium, including risk factors and prodromal symptoms.
Methods This study included sixty-five patients aged 65 years or older who had undergone hip surgery. Baseline assessments included age; gender; admission type (acute/elective); reason for surgery (fracture/replacement); C-reactive protein (CRP); Acute Physiology, Age, Chronic Health Evaluation (APACHE III); and the Mini-Mental State Examination (MMSE). The Korean version of the Delirium Rating Scale-Revised-98 (K-DRS-98) was used to assess prodromal symptoms daily before the onset of delirium.

Results Almost $28 \%(n=18)$ of the 65 patients developed delirium after surgery. Delirium in elderly patients after hip surgery was observed more often in older patients and those with acute admission, hip fracture, higher APACHE III score, lower MMSE score, and higher CRP levels within early days after the operation. Sleep-wake cycle disturbances, thought process abnormalities, orientation, and long-term memory in symptom items of K-DRS-98 were showed significant difference on 4 days before delirium, lability of affect on 3 days before, perceptual disturbances and hallucination, and visuo-spatial ability on 2 days before, and delusion, motor agitation, and short-term memory on the day before the occurrence of delirium. CRP levels within 24 hours and 72 hours after hospitalization were significantly higher in the delirium group.

Conclusion Medical professionals must pay attention to behavioral, cognitive changes and risk factors in elderly patients undergoing hip surgery and to the prodromal phase of delirium. K-DRS-98 may help in identifying the prodromal symptoms of delirium in elderly patients after hip surgery.

Psychiatry Investig 2011;8:340-347
\end{abstract}

Key Words Delirium, Elderly, Hip surgery, Prodromal, K-DRS-98.

\section{INTRODUCTION}

The incidence rates of delirium among all hospitalized patients vary widely from $6-56 \%$, and overall prevalence of delirium in the setting of general hospital admission this increases to $14-24 \% .{ }^{1}$ However, these incidence rates show a tendency to increase in elderly patients aged 65 and older, among male patients and patients with dementia or cognitive deficits, postoperative patients, and in patients treated in intensive care units. ${ }^{1}$ Researchers have reported that delirium occurs in 15-53\% of elderly patients aged 65 and older after a surgical operation, and in particular, the incidence rates of delirium in elderly patients after hip joint surgery vary widely,

Received: November 26, 2010 Revised: August 5, 2011

Accepted: August 5, 2011 Available online: December 8, 2011

$\triangle$ Correspondence: Jeong Lan Kim, MD, PhD

Department of Psychiatry, School of Medicine, Chungnam National University, 33 Munwha-ro, Jung-gu, Daejeon 301-721, Korea

Tel: +82-42-280-7287, Fax: +82-42-280-7886, E-mail: kimjl@cnu.ac.kr

(a) This is an Open Access article distributed under the terms of the Creative Commons Attribution Non-Commercial License (http://creativecommons.org/licenses/by$\mathrm{nc} / 3.0$ ) which permits unrestricted non-commercial use, distribution, and reproduction in any medium, provided the original work is properly cited. from $16-62 \%{ }^{2,3}$ Postoperative delirium in elderly patients represents a dangerous complication associated with high morbidity and mortality rates that requires prolonged hospitalization, thereby incurring additional physical, mental, and economic hardships to patients. About $30-40 \%$ of delirium can be prevented, and prevention of delirium is the most effective strategy for minimizing its occurrence and reducing related adverse results. ${ }^{4}$ Therefore, since the prevention and early treatment of delirium are important for the prognosis of the disease, interest in the prodromal stage of delirium has increased.

Although early symptoms of delirium develop in the prodromal stage before the diagnostic criteria are fully met, its accurate characteristics, incidence rates, and natural course are not well understood. In this period, changes in behaviors, emotions, and sleeping patterns are observed. ${ }^{5,6}$ Conceptually, early signs of delirium can be considered subsyndromal delirium, a syndrome not firmly diagnosed as delirium. ${ }^{7}$ One study defined subsyndromal delirium as including one or two of four areas: clouding of consciousness, reduced attention, disorientation, and cognitive deficits-the criteria for the diag- 
nosis of delirium. In this study, elderly hospitalized patients with subsyndromal delirium showed more delirium symptoms and lower levels of cognitive skills and functions in follow-ups compared to patients with no symptoms. ${ }^{8}$ Symptoms observed in the early period of delirium tend to be underdiagnosed because subsyndromal delirium or symptoms in the prodromal stage of delirium are not systematically known. Currently, even the DSM-IV does not include any distinction based on detailed phenomenological characteristics necessary for distinguishing subsyndromal delirium, and thus the necessity for related diagnostic criteria is being proposed for the DSM-V. ${ }^{9}$ Therefore, it is necessary to pay attention to early symptoms shown in the prodromal stage of delirium as such symptoms are quite meaningful, not only for the early diagnoses of delirium but also for subsyndromal delirium per se.

Delirium is a syndrome without a pathognomonic symptom or a specific laboratory test for diagnosis and the diagnosis relies only on clinical observation. ${ }^{10}$ However, in an attempt to discover biomarkers for delirium, several studies have looked at cytokine and C-reactive protein (CRP), which are inflammatory markers. CRP has been traditionally used as a marker of infection, inflammation and tissue damage-considered not only causes of but also the results of delirium. ${ }^{11}$ Previous studies reported that levels of CRP significantly increased in elderly patients with complications such as delirium and that CRP could be associated with a decline in cognitive performance. ${ }^{12,13}$ High CRP values, quite common in hospitalized elderly patients, are associated not only with depression and pain but also with dementia, thus, they are too non-specific to be considered as markers of delirium. ${ }^{14,15}$ Nevertheless, many studies have been conducted based on the premise that CRP could play an important role in monitoring the progress of delirium or in predicting recovery from delirium.

Several studies have attempted to identify symptoms in the prodromal stage using assessment tools for delirium. Researchers using the Neelon and Champagne (NEECHAM) confusion scale reported that early symptoms of delirium became aggravated 2-3 days after surgery, and observed reductions in NEECHAM scores from day 1 through day 4 after surgery. ${ }^{16,17}$ In a study conducted by de Jonghe et al, ${ }^{7}$ Delirium Rating Scale-Revised-98 (DRS-R-98) scores significantly increased below the cut-off point for diagnosis of delirium during the prodromal stage after surgery, before the occurrence of delirium and indicated that delirium could be predicted during the prodromal stage using memory impairment, incoherence, and disorientation symptoms. ${ }^{7}$ Compared to the importance of the prodromal stage of delirium, however, the characteristics in this period have not yet been positioned as a firm system, and thus, further studies are necessary for specific findings that must be considered for the early diagnoses of delirium in its prodromal stage, such as risk factors or behavioral, psychological, or cognitive changes.

In this respect, this study considers diagnostic strategic models helpful for early diagnoses and treatment of delirium by identifying diverse predictive factors through the examination of the preoperative cognitive and physical states; followup studies of CRP, assumed to be a biomarker of delirium; and assessments of prodromal-stage symptoms of delirium using the Korean version of the Delirium Rating Scale-Revised-98 (K-DRS-98) ${ }^{18}$ in elderly patients who will undergo hip joint surgery.

\section{METHODS}

\section{Subjects}

The subjects were patients of 65 years or older, hospitalized in the orthopedic surgery ward of Chungnam National University Hospital between March 2009 and February 2010. The procedures were explained to all participants and their families, and informed consent was received. Information was collected from the patients and their families through interviews with psychiatrists, and through medical records.

We excluded patients diagnosed with preoperative delirium; previously diagnosed with dementia or having previously shown severe cognitive impairment [Mini-Mental State Examination (MMSE) <10]; with a past history of delirium; taking anti-Parkinson drugs that may induce hallucinations; taking sedatives or cholinergic blocking agents that may cause delirium; and those with various other internal medicinal or surgical diseases and thus having systemic conditions severe enough to include the possibility of death. This study was approved by the Institutional Review Board of Chungnam National University Hospital (Approval number: 1003-26).

\section{Procedure}

Two steps made up the procedure: 1) preoperative early assessment to evaluate risks factors for delirium, and 2) prodromal stage assessment before and after surgery to identify the prodromal symptoms of delirium. In addition, we analyzed the CRP levels of the subjects at the completion of this study.

\section{Screening and initial assessments}

The initial assessment was completed within 24 hours after each elderly patient was hospitalized for hip joint surgery. Along with the assessment of age, a known risk factor for delirium, and risk factors that showed different results in studies such as gender, hospitalization types (emergency/planned), and purpose of surgery (fracture/substitution), in the initial assessment patients underwent Acute Physiology, Age, Chronic Health Evaluation III (APACHE III) ${ }^{19}$ for assessing physical 
severity and MMSE ${ }^{20}$ for assessing levels of cognitive skills.

\section{Prodromal stage assessment and the completion of assessments}

After screening and initial assessments, psychiatrists using K-DRS-98 conducted assessments daily before and after surgery until day 5 . The assessment time per case ranged from 5 to 10 minutes, depending on the patient. As mentioned, subjects in whom delirium occurred before surgery were excluded from the study, and when any patients had been diagnosed with delirium, treatment by psychiatrists began immediately without requesting consultation from the psychiatric department. Delirium was diagnosed using DSM-IV and K-DRS98. Patients who did not develop delirium due to anesthetic states that may have sustained until 6 hours after surgery were monitored until day 5 after surgery, and were included in all assessments. After the completion of the study, subjects were divided into two groups: those who developed delirium (delirium group) and those who did not develop delirium (nodelirium group). We then compared the results between the two groups.

\section{CRP follow-up}

Upon hospitalization in the orthopedic surgery ward for hip joint surgery, patient CRP was examined within 24 hours through blood tests. CRP was measured four times in total thereafter, including within 24 hours after surgery, 2-3 days after surgery, and 7 days after surgery. CRP is essentially examined during the hospitalization of any patient in the orthopedic department to monitor patient progress, and this study used examination results after getting agreement from patients and their families.

\section{Assessment tools}

\section{K-DRS-98}

Korean version of the Delirium Rating Scale-98 (K-DRS98), ${ }^{18}$ the Korean version of DRS-R-98, is a scale for assessing delirium. It is composed of 13 severity items and 3 diagnostic items. Each item is rated by the severity of the symptoms based on specific criteria. The severity items can be rated between 0 and 3 points, and the diagnostic items can be rated between 0 and 3 points. The highest score for the 16 items is 46 points, and the highest score for the severity items is 39 points. The cut-off of the severity score and the total score of the K-DRS-98 are 16 points and 21.5 points, respectively.

\section{MMSE-K}

Korean version of the Mini-Mental State Examination (MMSE-K), ${ }^{20}$ a simple and standardized tool, is the most wi- dely used measurement for cognitive functions. The MMSE$\mathrm{K}$, developed to fit Korean circumstances, even allows for illiterate patients. The maximum score is 30 points.

\section{APACHE III}

Acute Physiology, Age, Chronic Health Evaluation III (APACHE III) ${ }^{19}$ is a tool used to evaluate the severity of critically ill patients, and has a score distribution of 0 to 299 points. The higher the score, the worse the prognosis. The evaluation comprises three parts, including physiologic parametric scores ranging from 0 to 252 points, age scores ranging from 0 to 24 points, and comorbid disease scores ranging from 0 to 23 points. The physiologic parameters include pulse, mean blood pressure, body temperature, respiratory rate, $\mathrm{PaO}_{2}$, $\mathrm{AaDO}_{2}$, Hematocrit, white blood cell count, serum creatinine in patients with acute renal failure, serum creatinine in patients with no acute renal failure, daily total urine volume, blood urea nitrogen, sodium, albumin, bilirubin, blood sugar, neurologic anomalies, and acid-based disorders.

\section{Statistics}

Statistical analyses of all the data were conducted with the SAS 9.2 version.. Demographic characteristics and the characteristics of risk factors for delirium were compared two groups using t-tests and chi-square tests. A statistical signifi-

Table 1. Characteristics and risk factors of patients with delirium and patients without delirium

\begin{tabular}{lccc}
\hline \multicolumn{1}{c}{ Variable } & Delirium & No delirium & p value \\
\hline Patients (N=65) & $18(27.7 \%)$ & $47(72.3 \%)$ & \\
Age (Range) & $69-94$ & $65-90$ & \\
Age (mean \pm SD) & $81.7 \pm 6.35^{*}$ & $75.0 \pm 7.83$ & 0.002 \\
Male (N=22)/ & $8 / 10$ & $14 / 33$ & 0.380 \\
$\quad$ Female (N=43) & & & \\
Type of admission & $17 / 1^{* *}$ & $29 / 18$ & 0.013 \\
$\quad$ Acute/Elective) & & & \\
Type of Surgery & $17 / 1^{* *}$ & $28 / 19$ & 0.007 \\
$\quad$ Facture/Replacement) & & & \\
APACHE III (Mean \pm SD) & $36.6 \pm 7.15^{*}$ & $25.6 \pm 8.46$ & 0.000 \\
MMSE (Mean $\pm S D)$ & $16.6 \pm 2.64^{*}$ & $21.7 \pm 4.42$ & 0.000 \\
\hline
\end{tabular}

N: number, SD: standard deviation, APACHE III: Acute Physiology, Age, Chronic Health Evaluation III, MMSE: Mini-Mental State Examination

Table 2. Onset of delirium in patients after hip surgery

\begin{tabular}{ccccc}
\hline & $\begin{array}{c}\text { Day } \\
\text { of Surgery }\end{array}$ & $\begin{array}{c}\text { Second } \\
\text { day }\end{array}$ & $\begin{array}{c}\text { Third } \\
\text { day }\end{array}$ & $\begin{array}{c}\text { Fourth } \\
\text { day }\end{array}$ \\
\hline $\begin{array}{c}\text { Delirium } \\
(\mathrm{N}=18)\end{array}$ & $7(38.9 \%)$ & $7(38.9 \%)$ & $3(16.7 \%)$ & $1(5.5 \%)$ \\
\hline $\mathrm{N}$ number & & & &
\end{tabular}


cance level was $\mathrm{p}<0.05$. Symptom items of K-DRS- 98 on 4 days before, 3 days before, 2 days before and one day before delirium diagnosis were compared in delirium group and No delirium group using independent t-test. For each symptom item of K-DRS-98, repeated-measure ANOVA of general linear model was performed to test the effects of the occurrence of delirium (between-group factor) and time (within group factor).

\section{RESULTS}

\section{Characteristics and risk factors for the}

\section{delirium group and the no-delirium group}

Of 65 patients, 27.7\% ( $\mathrm{n}=18)$ developed delirium after hip joint surgery. The mean age of the delirium group was $81.7 \pm 6.4$ years, significantly higher than the mean age of the no-delirium group ( $75.0 \pm 6.4$ years). Of the 65 study subjects, 22 were male and 43 were female, and gender did not show any statistical significance between the two groups. In both groups, acute admission due to hip fracture was more frequent than patients admitted for elective surgery. In terms of types of surgery, the incidence rate of delirium was significantly higher in cases where the patients were hospitalized due to a fracture rather than for replacement of hip. The delirium group had higher scores on the APACHE III compared to the no-delirium group. On the other hand, the delirium group demonstrated significantly lower scores on the MMSE (16.6 \pm 2.64$)$ compared to the no-delirium group (21.7 \pm 4.42$)$ (Table 1).

Of 18 patients diagnosed with delirium after surgery, 7 (38.9\%) received the diagnosis on day 1 after surgery, 7 $(38.9 \%)$ on day 2 after surgery, $3(16.7 \%)$ on day 3 after surgery, and 1 (5.5\%) on day 4 after surgery (Table 2 ).

\section{Prodromal stage symptoms of delirium}

Symptom items of K-DRS-98 on 4 days before, 3 days be-
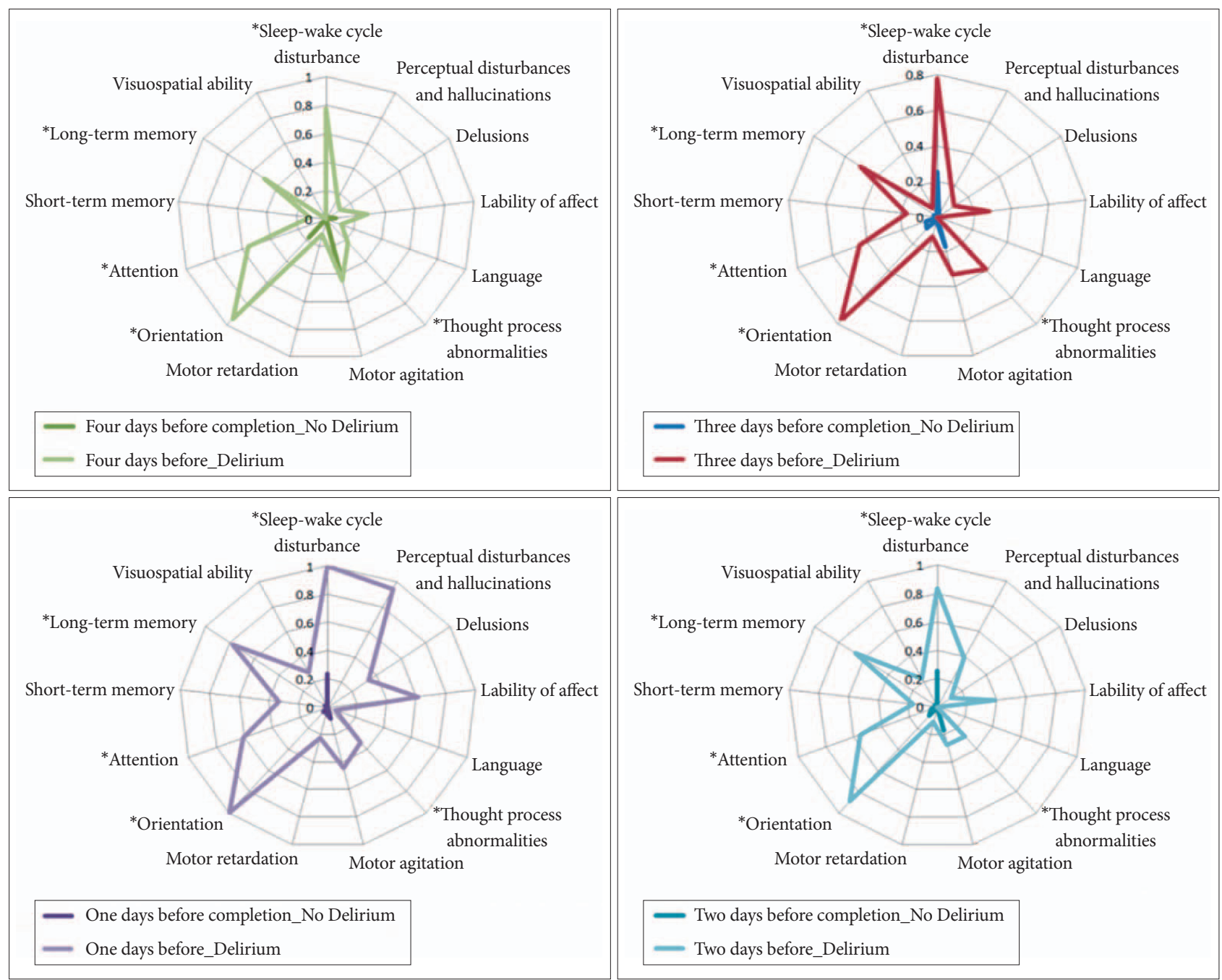

Figure 1. Comparison of symptom items of K-DRS-98 between Delirium group and No-Delirium group by Radar Chart. K-DRS-98: Korean version of the Delirium Rating Scale-Revised-98. ${ }^{*} \mathrm{p}<0.05$. 
fore, 2 days before and one day before delirium diagnosis in the delirium group were compared with those of corresponding days in no- delirium group using independent t-test. Figure 1 shows the differences of average score of 13 symptom items of K-DRS-98 in prodromal stage between the delirium group and the no-delirium group. Sleep-wake cycle disturbances, thought process abnormalities, orientation, and longterm memory were showed significant difference on 4 days before delirium in delirium group, and then lability of affect on 3 days before, perceptual disturbances and hallucination, and visuo-spatial ability on 2 days before, and delusion, motor agitation, and short-term memory on one day before additionally, compared no- delirium group. In the prodromal stage, symptoms were getting increase over time, and most symptoms, except of motor retardation and language were showed significant difference in the delirium group compared the no-delirium group. While the averages total K-DRS-98 score of the no-delirium group of each assessment day were not changed, , the delirium group showed an average score of $5.56 \pm 1.34$ for 4 days before delirium diagnosis, $5.56 \pm 2.043$ days before, $7.22 \pm 2.29$ for 2 days before and $9.56 \pm 3.24$ for 1 day before delirium diagnosis. Repeated-measures ANOVA revealed significant differences between two groups in symptoms of K-DRS-98 (Table 3). In addition, most symptoms of K-DRS-98, except sleep-wake cycle disturbances, attention, and physical disorder, were changed over time in the delirium group.

\section{Changes in CRP over time}

Within 24 hours after hospitalization, the delirium group showed higher CRP levels compared to the no-delirium group, but the differences were not statistically significant. CRP measured within 24 hours after hospitalization showed the highest levels among four measurements in both groups and the CRP levels demonstrated a tendency to decrease thereafter. CRP levels measured within 24 hours after hospitalization and those measured within 72 hours after hospitalization were shown to be statistically significantly higher in the delirium group compared to the no-delirium group. No statistically significant differences in CRP values 7 days after surgery were observed between the two groups (Figure 2).

\section{DISCUSSION}

This study was aimed to identify the occurrence of delirium after hip joint surgery, by examining the preoperative physical states of elderly patients who undergo hip joint surgery, examining CRP as a biomarker of delirium before surgery, following up on CRP postsurgery, and assessing patients' cognitive and behavioral symptoms during the prodromal stage of delirium using the K-DRS-98.

As expected, and in agreement with other studies, in this study, delirium showed a tendency to occur in older elderly patients who underwent hip joint surgery in the case of emergency hospitalization, surgery due to fractures rather than

Table 3. Analyses for effects of group and day on symptoms of K-DRS-98

\begin{tabular}{|c|c|c|c|c|c|c|}
\hline & \multicolumn{2}{|c|}{ Group } & \multicolumn{2}{|c|}{ Day } & \multicolumn{2}{|c|}{ Group $\times$ day } \\
\hline & F value & p-value & F value & p-value & F value & p-value \\
\hline Sleep-wake cycle disturbance & 38.72 & $<0.0001$ & 2.37 & 0.0796 & 3.94 & 0.0123 \\
\hline Perceptual disturbances and hallucinations & 28.69 & $<0.0001$ & 18.52 & $<0.0001$ & 18.89 & $<0.0001$ \\
\hline Delusions & 22.78 & $<0.0001$ & 6.62 & 0.0025 & 7.15 & 0.0016 \\
\hline Lability of affect & 71.58 & $<0.0001$ & 5.65 & 0.0017 & 7.39 & 0.0003 \\
\hline Language & 2.68 & 0.1066 & 4.48 & 0.0152 & 4.48 & 0.0152 \\
\hline Thought process abnormalities & 22.78 & $<0.0001$ & 5.42 & 0.0023 & 5.42 & 0.0023 \\
\hline Motor agitation & 13.06 & 0.0006 & 3.29 & 0.0264 & 4.47 & 0.0067 \\
\hline Motor retardation & 5.27 & 0.0251 & 2.77 & 0.0704 & 1.36 & 0.2651 \\
\hline Orientation & 127.08 & $<0.0001$ & 4.34 & 0.0077 & 3.95 & 0.0122 \\
\hline Attention & 71.58 & $<0.0001$ & 0.81 & 0.4944 & 2.82 & 0.0466 \\
\hline Short-term memory & 22.78 & $<0.0001$ & 4.13 & 0.0207 & 4.13 & 0.0207 \\
\hline Long-term memory & 114.74 & $<0.0001$ & 4.32 & 0.008 & 4.32 & 0.008 \\
\hline Visuospatial ability & 17.52 & $<0.0001$ & 5.65 & 0.0017 & 0.65 & 0.0017 \\
\hline Temporal onset of symptoms & 223.92 & $<0.0001$ & 3.47 & 0.0213 & 6.22 & 0.0009 \\
\hline Fluctuation of symptoms severity & 227.77 & $<0.0001$ & 73.51 & $<0.0001$ & 73.51 & $<0.0001$ \\
\hline Physical disorder & 363.36 & $<0.0001$ & 2.57 & 0.0622 & 7.95 & 0.0001 \\
\hline
\end{tabular}

K-DRS-98: Korean version of the Delirium Rating Scale-Revised-98 


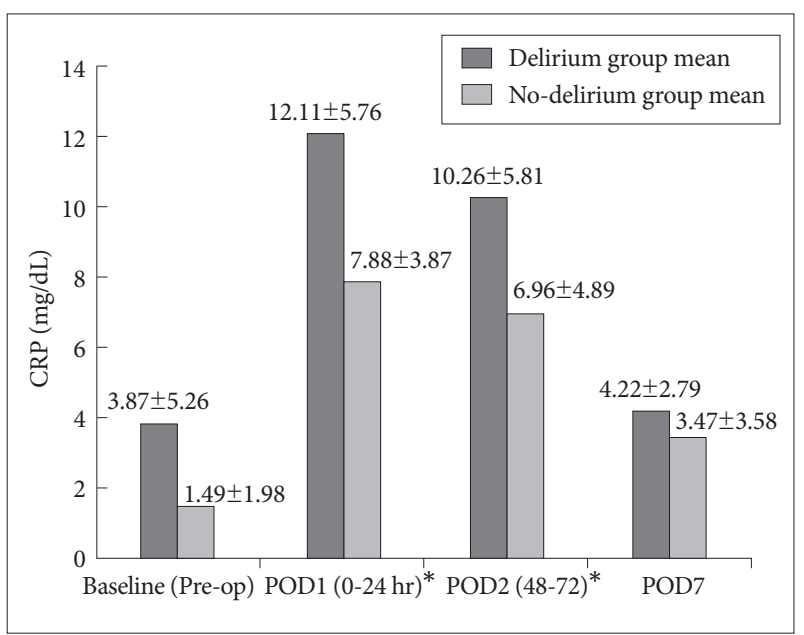

Figure 2. CRP in delirium patients after hip surgery, compared to no-delirium patients. CRP: C-reactive protein, POD: postoperative day. ${ }^{*} p<0.05$, t-test.

substitution, ${ }^{7,21}$ higher APACHE III scores, and lower MMSE scores. Although males are classified as having a high risk for the occurrence of delirium, ${ }^{1}$ our study revealed no difference in the occurrence of delirium between genders. Patients who developed delirium showed higher APACHE III scores and lower MMSE scores, and the frequency of the occurrence of delirium was higher with the higher severity of physical disease and more severe cognitive decline. A recent study by Franco et $\mathrm{al}^{22}$ showed the correlation with MMSE score at admission and risk for delirium in older medical inpatients. They suggested that optimal MMSE cutoff score was 24.5. In our study, we included all subjects, who has over 10 on MMSE and were not met dementia of DSM-IV. Therefore, the cognitive impairment can be affected on the occurrence of delirium.

In this study, delirium occurred most frequently on day 1 and day 2 after surgery at the same rate, but the rate of the occurrence of delirium until day 3 after surgery was $94.5 \%$ of the entire cases of delirium, indicating that most cases of delirium occur between days 1 and 3 after surgery. This result is consistent with the result of a study by Lemstra ${ }^{23}$ who reported that it took 1.5 days after surgery on average for delirium to occur and the results of studies by Santos et al..$^{24}$ and de Jonghe et al. ${ }^{7}$ who reported that delirium occurred most frequently on days 2 and 3 after surgery.

We assessed the cognitive and behavioral changes during the prodromal stage of delirium using K-DRS-98. The items, sleep-wake cycle disturbances, thought process abnormalities, orientation, attention, and long term memory of K-DRS-98 appeared first and continued for a long time. Then, lability of affect was observed, then perceptual disturbances and hallucination and visuospatial ability were observed, followed by delusisons, motor agitation, and short-term memory on the day before the occurrence of delirium. Data demonstrated that the number of symptoms increased during the prodromal stage of delirium as the date of occurrence of delirium neared, and symptom severity scores also increased. The temporal onset of symptoms and the existence of physical symptoms, diagnostic items for delirium, could support the phenomenological symptoms shown during the prodromal stage of delirium as ground items for diagnosis of delirium later. Duppils et al. ${ }^{25}$ reported anxiety and disorientation during the prodromal stage of delirium in elderly patients who developed delirium after undergoing hip joint surgery, and de Jonghe et al. ${ }^{7}$ reported memory disorder, incoherence, and disorientation as prodromal symptoms of delirium. Our results were generally consistent with the results of previous studies except for the addition of affective instability, which can be seen as a difference.

As with previous experiments, predicting delirium with preoperative CRP levels proved difficult, but the levels within 24 hours after surgery and within 48-72 hours after surgery showed statistically significant differences in the delirium group. These periods correspond to the prodromal stage of delirium when changes in cognitive skills and behaviors began to appear. However, the level of CRP was not measured on the same day of assessment of K-DRS-98 in all subjects. Therefore we did not analyze the correlation with the level of CRP and symptoms severity of K-DRS-98. Given the results of studies by Beloosesky et al. ${ }^{26}$ and Macdonald et al. ${ }^{27}$ that showed an association between increased CRP levels in elderly patients who underwent hip joint surgery and cognitive skill decline, CRP level increases in the prodromal stage can be considered a marker for early diagnoses of delirium. However, the study by Beloosesky et al. ${ }^{26}$ did not use any assessment tool for delirium, and the study by Macdonald et al. ${ }^{27}$ only showed CRP level predict incident delirium. A prospective study for prodromal phase of delirium is needed to evaluate the value of CRP as a marker for early diagnosis of delirium in future.

As limitations of this study, the following should be considered. First, given the many exclusion criteria in screening the patients, some patients with the risk factor of delirium were not included, and thus, the incidence rates of delirium measured in this study may be lower than actual incidence rates. Second, although the K-DRS-98 is a well-known assessment tool with proven validity in measuring the severity of delirium, this scale cannot predict all other important prodromal symptoms. Third, the number of subjects was relatively small for generalizing the results of this study. Fourth, we do not use systematized and structured assessment tool for exclusion of dementia. This can be included cognitive impaired subjects, who has higher risk to develop delirium. 
In spite of its limitations, our study identified not only factors already known as risk factors for delirium, such as old age, cognitive skill decline, and declined physical condition, but also the fact that the possibility of the occurrence of delirium is particularly high in elderly patients who undergo hip joint surgery in the case of emergency hospitalization and fracture surgery. Some specific items of K-DRS-98, such as sleep-wake cycle disturbances, thought process abnormalities, orientation, attention, and long-term memory can be considered early symptoms of delirium in patients with risk factors. As Franco et al. ${ }^{22}$ showed the correlation with score of MMSE and DRS-R-98 score in delirium, a cognitive assessment can be predictive in patients with many risk factors. We could consider the possibility of using CRP as a biomarker of delirium along with clinical observation.

Gurlit et al. ${ }^{28}$ suggested that education on the risk factors of delirium and major clinical characteristics in the prodromal stage could play an important role in preventing and treating postoperative delirium. Identifying the risk factors of the occurrence of delirium is basic, and a screening test for cognitive functions is essential. The perception of early clinical signs should be helpful in the prevention and treatment of delirium and be able to bring about better prognoses.

In this study, to predict and treat delirium early in elderly patients who underwent hip joint surgery, we used the severity of a patient's physical symptoms before surgery, levels of cognitive skills, and behavioral and mental changes during the prodromal stage and follow-ups using CRP as a biomarker of delirium. Although this study may be considered as having excessively simplified the complicated multiple models of the etiology of delirium, the results of this study will help screening tests for delirium in high-risk patients at such as elderly patients after hip joint surgery. Based on the results of this study, an extended model can be considered as a screening test conducted through factors for predicting the occurrence of delirium in not only elderly patients who undergo hip joint surgery but also other populations at risk of delirium.

\section{Acknowledgments}

This study was financially supported by research fund of Chungnam National University in 2009.

\section{REFERENCES}

1. Fong TG, Tulebaev SR, Inouye SK. Delirium in elderly adults: diagnosis, prevention and treatment. Nat Rev Neurol 2009;5:210-220.

2. Inouye SK. Delirium in hospitalized older patients. Clin Geriatr Med 1998;14:745-764.

3. Bitsch MS, Foss N, Kristensen B, Kehlet H. Pathogenesis of and management strategies for postoperative delirium after hip fracture: a review. Acta Orthop Scand 2004;75:378-389.

4. Siddiqi N, House AO, Holmes JD. Occurrence and outcome of delirium in medical in-patients: a systematic literature review. Age Ageing 2006;35:350-364.
5. Meagher DJ, Trzepacz PT. Delirium phenomenology illuminates pathophysiology, management, and course. J Geriatr Psychiatry Neurol 1998;11:150-156.

6. Gupta N, de Jonghe J, Schieveld J, Leonard M, Meagher D. Delirium phenomenology: what can we learn from the symptoms of delirium? J Psychosom Res 2008;65:215-222.

7. de Jonghe JF, Kalisvaart KJ, Dijkstra M, van Dis H, Vreeswijk R, Kat $\mathrm{MG}$, et al. Early symptoms in the prodromal phase of delirium: a prospective cohort study in elderly patients undergoing hip surgery. Am J Geriatr Psychiatry 2007;15:112-121.

8. Cole M, McCusker J, Dendukuri N, Han L. The prognostic significance of subsyndromal delirium in elderly medical inpatients. J Am Geriatr Soc 2003;51:754-760.

9. Meagher D, Trzepacz PT. Phenomenological distinctions needed in DSM-V: delirium, subsyndromal delirium, and dementias. J Neuropsychiatry Clin Neurosci 2007;19:468-470.

10. American Psychiatric Association. Practice guideline for the treatment of patients with delirium. Am J Psychiatry 1999;156(5 Suppl):1-20.

11. Beloosesky Y, Grinblat J, Pirotsky A, Weiss A, Hendel D. Different Creactive protein kinetics in post-operative hip-fractured geriatric patients with and without complications. Gerontology 2004;50:216-222.

12. Tilvis RS, Kahonen-Vare MH, Jolkkonen J, Valvanne J, Pitkala KH, Strandberg TE. Predictors of cognitive decline and mortality of aged people over a 10-year period. J Gerontol A Biol Sci Med Sci 2004;59: 268-274.

13. Yaffe K, Kanaya A, Lindquist K, Simonsick EM, Harris T, Shorr RI, et al. The metabolic syndrome, inflammation, and risk of cognitive decline. JAMA 2004;292:2237-2242.

14. Liukkonen T, Silvennoinen-Kassinen S, Jokelainen J, Räsänen P, Leinonen M, Meyer-Rochow VB, et al. The association between C-reactive protein levels and depression: Results from the northern Finland 1966 birth cohort study. Biol Psychiatry 2006;60:825-830.

15. Graham JE, Robles TF, Kiecolt-Glaser JK, Malarkey WB, Bissell MG, Glaser R. Hostility and pain are related to inflammation in older adults. Brain Behav Immun 2006;20:389-400.

16. Hattori H, Kamiya J, Shimada H, Akiyama H, Yasui A, Kuroiwa K, et al. Assessment of the risk of postoperative delirium in elderly patients using E-PASS and the NEECHAM Confusion Scale. Int J Geriatr Psychiatry 2009;24:1304-1310.

17. Matsushita T, Matsushima E, Maruyama M. Early detection of postoperative delirium and confusion in a surgical ward using the NEECHAM confusion scale. Gen Hosp Psychiatry 2004;26:158-163.

18. Lim KO, Kim SY, Lee YH, Lee SW, Kim JL. A validation study for the Korean version of Delirium Rating Scale-Revised-98 (K-DRS-98). J Korean Neuropsychiatr Assoc 2006;45:518-526.

19. Knaus WA, Wagner DP, Draper EA, Zimmerman JE, Bergner M, Bastos PG, et al. The APACHE III prognostic system. Risk prediction of hospital mortality for critically ill hospitalized adults. Chest 1991; 100:1619-1636.

20. Folstein MF, Folstein SE, McHugh PR. "Mini-mental state”. A practical method for grading the cognitive state of patients for the clinician. J Psychiatr Res 1975;12:189-198.

21. Bruce AJ, Ritchie CW, Blizard R, Lai R, Raven P. The incidence of delirium associated with orthopedic surgery: a meta-analytic review. Int Psychogeriatr 2007;19:197-214.

22. Franco JG, Valencia C, Bernal C, Ocampo MV, Trzepacz PT, Pablo J, et al. Relationship between cognitive status at admission and incident delirium in older medical inpatients. J Neuropsychiatry Clin Neurosci 2010;22:329-337.

23. Lemstra AW, Kalisvaart KJ, Vreeswijk R, van Gool WA, Eikelenboom P. Pre-operative inflammatory markers and the risk of postoperative delirium in elderly patients. Int J Geriatr Psychiatry 2008;23:943-948.

24. Santana Santos F, Wahlund LO, Varli F, Tadeu Velasco I, Eriksdotter Jonhagen M. Incidence, clinical features and subtypes of delirium in elderly patients treated for hip fractures. Dement Geriatr Cogn Disord 
2005;20:231-237.

25. Duppils GS, Wikblad K. Delirium: behavioural changes before and during the prodromal phase. J Clin Nurs 2004;13:609-616.

26. Beloosesky Y, Hendel D, Weiss A, Hershkovitz A, Grinblat J, Pirotsky A, et al. Cytokines and C-reactive protein production in hip-fracture-operated elderly patients. J Gerontol A Biol Sci Med Sci 2007;62:420-426.
27. Macdonald A, Adamis D, Treloar A, Martin F. C-reactive protein levels predict the incidence of delirium and recovery from it. Age Ageing 2007;36:222-225.

28. Gurlit S, Möllmann M. How to prevent perioperative delirium in the elderly? Z Gerontol Geriatr 2008;41:447-452. 